\title{
Cinemática en el laboratorio de física: encuentro entre dos móviles con Movimiento Rectilíneo Uniforme.
}

María Luz Meza Borda Dansey; Saúl E. Torres Ercolani; César A. Cabrera; Carlos A. Merino; Patricio F. Provasi; Erika N. Bentz( $\left.{ }^{*}\right)$

\section{Resumen:}

Normalmente un curso de física general, tanto en el nivel medio como universitario, tiene como punto de partida la cinemática, lo que se refleja en la mayoria de los libros de texto y es el comienzo de los contenidos para la enseñanza y aprendizaje del resto de la fisica. Por esta razón decidimos diseñar un experimento para el laboratorio que pueda funcionar como complemento o inicio de una clase para luego pasar a la explicación formal en el pizarrón.

Este experimento puede fabricarse con materiales suficientemente básicos y económicos como para ser desarrollado por alumnos de asignaturas del nivel superior de la carrera de física y utilizado con poco más que la intuición por alumnos del primer curso con resultados suficientemente precisos.
Palabras clave: Cinemática; Movimiento Rectilíneo Uniforme (MRU); dispositivo de medida; material didáctico.

Title: Kinematics in the physics laboratory: meeting between two mobiles with Uniform Rectilinear Movement.

Abstract: Normally a general physics course, both at the middle and university level, has as its starting point the kinematics, which is reflected in most textbooks and is the beginning of the content for teaching and learning the rest of physics. For this reason, we decided to design an experiment for the laboratory that could work as a complement or start of a class and then move on to the formal explanation on the blackboard.

This experiment can be made with materials that are sufficiently basic and economical to be developed by students 
of subjects of the higher level of the physics degree and used with little more than intuition by students of the first year with sufficiently precise results.

\section{Keywords: Kinematics; $\quad$ URM;} measurement device; classroom material

\section{Introducción}

Las dificultades que encuentran los alumnos de los últimos años del nivel secundario y los primeros años del nivel universitario para comprender los conceptos de cinemática han sido estudiadas a lo largo de las últimas décadas y aún es un tema abierto al análisis y debate. En particular muchos de estos estudios se centran en la dificultad de los estudiantes en comprender las ecuaciones de la cinemática y la interpretación de los gráficos que se obtienen a partir de ellas (Sathe, (1979); Guidugli y otros (2005); Nordine, (2011); Kagan, (2013); Berryhill y otros (2016); Vučeljić, Šuškačević, (2016); De Luca, Faella (2017); Klein, $y$ otros (2017)). En algunos casos con soluciones muy ingeniosas y la mayoria bastante atractivas a la hora de Llamar la atención de los estudiantes o evaluar su aprendizaje. En todos ellos el proceso enseñanza-aprendizaje está dentro del marco del pizarrón y/o el libro de apuntes del estudiante. Sin embargo una cantidad no menos significativa de trabajos involucra la experimentación en el laboratorio como demostración en el proceso enseñanza-aprendizaje (Stroink y otros (2003); Bartlett, (2005); Nelson, (1983); Rich, (1989); Falcão y otros (2009); Lowe, Ayars (2010); Kranjc, Razpet, (2011); Gabriel, Backhaus, (2013)).

La cinemática es un tópico, entre otros, en que un estudiante es consciente en el sentido que lo usa normalmente en la vida cotidiana, pero nunca lo vio formalmente hasta el final de la escuela secundaria o primer año de la universidad. Diariamente observa el movimiento de las cosas y hasta es capaz de predecir la trayectoria de un objeto, de forma de atraparlo (una pelota, un compañero de juegos) o esquivarlo (una piedra, un automóvil). Como sea, esto ocurre independientemente de la formalidad matemática y fuera de un salón de clases, y por lo tanto el joven construye su propio entendimiento de la cinemática. Así, éste conocimiento basado en el empirismo del día a día no siempre resulta sencillo de corregir bajo la luz de la física formal. Esto ocurre, normalmente debido a que la mente genera por cada hipótesis una tesis que puede o no ser la correcta, así alguien puede Llenarse de prejuicios que redunda en una tarea muy difícil, sino imposible, para el profesor de física ya que normalmente estos prejuicios vienen acompañado de un manejo muy básico, y a veces incompleto, de las matemáticas. Sin embargo es sabido que la enseñanza se consuma cuando el significado del material que el alumno 
capta es el significado que el profesor pretende que ese material tenga para el alumno (Gowin, D. B. (1981)).

En este trabajo desarrollamos un dispositivo para el laboratorio con el cual se puede resolver un problema de encuentro entre dos partículas con movimientos rectilineos uniformes (MRU). Hasta el presente tales dispositivos no abundan, y los pocos que se pueden encontrar, para tal fin, no son de simple realización y en general su costo es más bien elevado.

El diseño de este dispositivo se pensó con el propósito de crear una experiencia de laboratorio de física en el tema de cinemática de forma que los estudiantes puedan observar por si mismos, en un entorno experimental controlado y accesible, una de las experiencias cotidianas más simples del día a día, y mediante el empleo de las ecuaciones del MRU poder predecir el lugar de encuentro entre dos móviles que se dirigen uno hacia el otro. Se busca que la metodología empleada le permita al alumno experimentar, describir, analizar y valorar el proceso.

\section{Materiales y método}

En general uno de los desafíos del docente de física es definir contextos para la instrucción que motive a los estudiantes y capture su atención. Esta propuesta permite a los estudiantes tener una enseñanza práctica evitando la aplicación de una metodología tradicional donde el estudiante es un sujeto pasivo y el docente se convierte en un expositor de conocimiento.

El presente trabajo muestra el diseño y aplicación de una propuesta de intervención en el Laboratorio de la cátedra que busca propiciar en los estudiantes la adquisición de los conceptos de la cinemática del movimiento rectilíneo por medio de la experimentación.

En este contexto, se hace necesario que los recursos que se usen para apoyar el proceso de enseñanza de la física, así como el de cualquier ciencia natural, no tengan solo el propósito de ilustración, sino que deben permitir la interacción y ayudar a la construcción de representaciones internas complejas; que permitan a los estudiantes explicar y predecir fenómenos en un proceso donde jueguen un papel de observadores activos.

El dispositivo consta de un tubo de vidrio de aproximadamente $1 \mathrm{~cm}$ de diámetro, sellado en ambos extremos y lleno con un fluido viscoso transparente (agua, vaselina, glicerina, o aceite en general), no inflamable de ser posible. Los móviles están representados por una bolita de acero de aproximadamente $0.5 \mathrm{~cm}$ de diámetro y una burbuja de aire de aproximadamente el mismo 
diámetro que la bolita. El tubo de vidrio se encuentra recubierto por un tubo metálico con un diámetro ligeramente superior al primero y se encuentra adherido a una base metálica apoyada en un soporte metálico que permite variar la inclinación del tubo y trabajar asi con distintas pendientes (ver figura 1). Sobre el lateral del tubo de vidrio hay una escala graduada en milímetros de $50 \mathrm{~cm}$ con un margen de $6 \mathrm{~cm}$ en cada extremo del tubo. Se busca que los móviles se desplacen con constante.

El dispositivo también cuenta con un imán de neodimio lo suficientemente potente como para poder atraer a la bolita de acero y retenerla en el extremo superior, debajo de las capas metálicas, aunque también se podrían reemplazar las capas metálicas por estructuras plásticas.

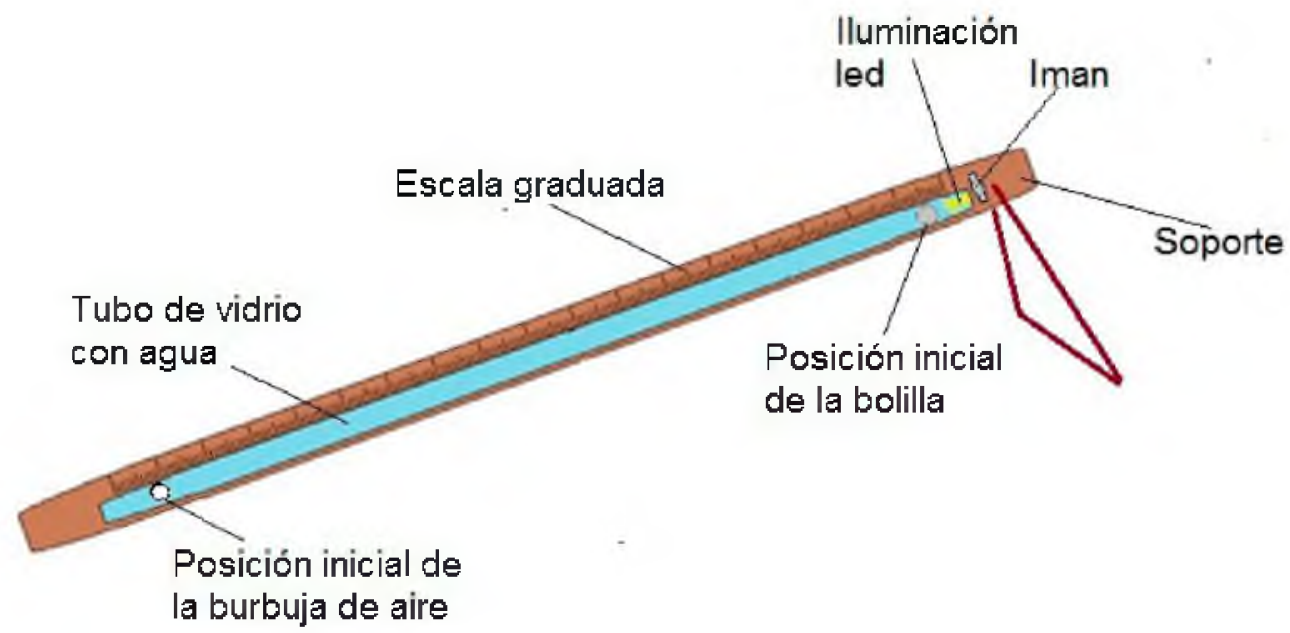

FIGURA 1. Se muestra un esquema del dispositivo propuesto para el laboratorio de física en el desarrollo del tema encuentro de dos móviles encuadrado en cinemática

\section{Funcionamiento:}

Para la realización del experimento, hay que estar familiarizado con el MRU, lo que normalmente ocurre, si se está habituado al movimiento unidimensional con aceleración constante (MRUA) y considerando al primero como un caso

$$
a \quad k \quad y \quad v=v_{0}+a \cdot t \quad r=r_{0}+v_{0} \cdot t+\frac{1}{2} \cdot a \cdot t^{2}
$$


que, para el caso particular en que $\mathrm{k}=0$; se reducen a un MRU

$$
a=0 \quad ; \quad y=v_{0} \quad y \quad y_{0}^{*}+v_{0} \cdot r
$$

La medición de las velocidades medias de la bolita y de la burbuja se realiza con la ayuda de la escala graduada que está junto al tubo de vidrio y un cronómetro usualmente, el teléfono móvil. Así se tiene

$$
v^{\text {boliilla }}=\frac{\Delta x^{\text {bolita }}}{\Delta t^{\text {bolita }}} \text { y } v^{\text {burbuja }}=\frac{\Delta x^{\text {burbuja }}}{\Delta t^{\text {burbuja }}}
$$

Para resolver el problema de encuentro que se plantea en este laboratorio debemos determinar en qué momento se encontrarán los dos móviles (la burbuja y la bolita de acero) que se desplazan en sentidos opuestos describiendo movimientos rectilíneos uniformes; para ello realizamos el siguiente análisis:

Ya que la burbuja parte de la posición inferior del tubo y la bolita de acero desde la superior al momento de remover el imán tenemos:

$$
r^{\text {burbuja }}=r_{0}^{\text {burbuja }}+v_{0}^{\text {burbuja }} \cdot t \quad \text { y } \quad r^{\text {bolita }}=r_{0}^{\text {bolita }}-v_{0}^{\text {bolita }} \cdot t
$$

Considerar que el origen de coordenadas se ubica en la parte inferior.

Sabiendo que $r_{0}^{\text {burbuja }}=0 \mathrm{~cm}$ y que $r_{0}^{\text {bolita }}=50 \mathrm{~cm}$ y que el encuentro ocurrirá cuando $r^{\text {burbuja }}=r^{\text {bolita }}$, podemos deducir que:

$$
t=\frac{-r_{0}^{\text {burbuja }}+r_{0}^{\text {bolita }}}{v_{0}^{\text {burbuja }}+v_{0}^{\text {bolita }}}
$$

Entonces reemplazando este valor de $t$ en cualquiera de las ecuaciones (4) obtenemos

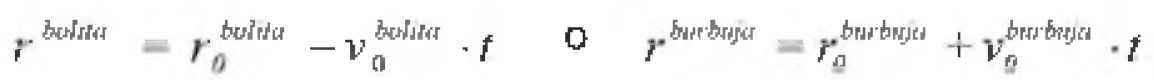


Todo el procedimiento se realiza filmando el dispositivo en una misma toma que incluya al cronómetro. Tanto filmadora como cronómetro; pueden ser teléfonos móviles.

\section{Punto de encuentro:}

1) Con ayuda de los fundamentos teóricos analizar el caso de la bolita que cae y la burbuja que sube para determinar analíticamente en qué punto del recorrido se encontrarán, si es que comienzan a desplazarse en el mismo instante inicial. Realizar los cálculos considerando que las velocidades de los móviles son los promedios hallados anteriormente.

2) Comprobar que la burbuja se encuentra en la parte baja del sistema y llevar la bolita a la parte alta con ayuda del imán. Para el mismo ángulo estudiado anteriormente; comprobar experimentalmente el punto del camino en el que tiene lugar el encuentro.

Aclaración: Como el procedimiento fue filmado, se puede llevar la filmación hasta el momento en que la burbuja está en el $\mathrm{o} \mathrm{cm}$ de la escala y el tiempo indicado por el cronómetro será el que hay que descontar a todas las demás marcas temporales.

Cuando la burbuja coincide con el o cm, generalmente la bolita que se mueve a una velocidad diferente; no se encuentra en $50 \mathrm{~cm}$ de la misma escala, pero de la filmación se puede ver la posición inicial y es la que luego se va a usar como $r_{0}^{\text {boina }}$. En el caso de la experiencia realizada en este trabajo el valor es, $r_{i p}^{\text {butilua }}=51 \mathrm{~cm}$

3) Comparar resultados y realizar conclusiones.

Resultados:

a) Promedio de velocidades (para la burbuja que sube):

$$
v^{\text {burbuja }}=\frac{\sum_{i=1}^{N} v_{i}^{\text {burbuja }}}{N}
$$


b) Promedio de velocidades (para la bolita de acero):

$$
v^{\text {bolita }}=\frac{\sum_{i=1}^{N} v_{i}^{\text {boitina }}}{N}
$$

c) Punto de encuentro (hallado experimentalmente): El estudiante confecciona los gráficos de los móviles y obtiene dos líneas rectas que se cruzan en el punto que corresponde al lugar donde los móviles se encuentran.

d) Punto de encuentro (hallado analíticamente):

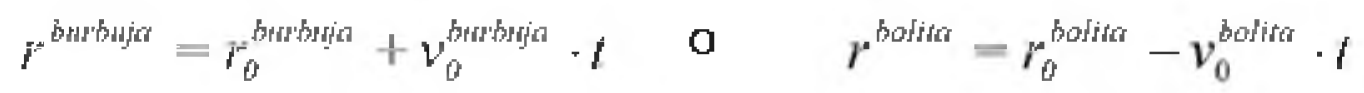

e) Conclusiones y reflexiones finales: A cargo del alumno.

Para el caso particular del experimento llevado a cabo en el desarrollo de este trabajo, los resultados obtenidos son los siguientes:

TABLA I. Valores de tiempo expresado en segundos y velocidad expresada

\begin{tabular}{|c|c|c|c|c|c|c|c|c|c|}
\hline \multicolumn{5}{|c|}{ BUREUJA } & \multicolumn{5}{|c|}{ BOLITADE ACERO } \\
\hline $\begin{array}{c}\text { Espacio } \\
\text { [m] }\end{array}$ & $\Delta r^{\prime}$ & $\begin{array}{c}\text { Tie mpo } \\
\text { (step) }\end{array}$ & $\Delta l^{*}$ & $\begin{array}{l}\text { Welocidad } \\
\text { (omseg, }\end{array}$ & $\begin{array}{c}\text { Espacio } \\
\text { [m, }\end{array}$ & $\Delta r^{\prime \prime}$ & $\begin{array}{c}\text { Trempo } \\
\text { (seg) }\end{array}$ & $A t^{\circ}$ & $\begin{array}{l}\text { Welocidad } \\
\text { (ont'seg) }\end{array}$ \\
\hline 0 & & 6.3 .1 & & & 51 & & 6.59 & & \\
\hline 10 & 10 & 8.65 & 2.34 & 4.27 & 40 & 10 & 10.58 & 3.99 & 2.51 \\
\hline 20 & 10 & 11.07 & 2.42 & 4.19 & 30 & 10 & 14.45 & 3.87 & 2.58 \\
\hline 30 & 10 & 13.54 & 2.47 & 4.05 & 20 & 10 & 18.38 & 3.93 & 2.54 \\
\hline 40 & 10 & 15.86 & 2,32 & 4.31 & 10 & 10 & 22.40 & 4.02 & 2,49 \\
\hline 50 & 10 & 18.38 & 2.52 & 3.97 & 0 & 10 & 26.56 & 4.16 & 2,40 \\
\hline
\end{tabular}
en centimetros/segundos para los dos móviles

'A hace referencia a interwalos 


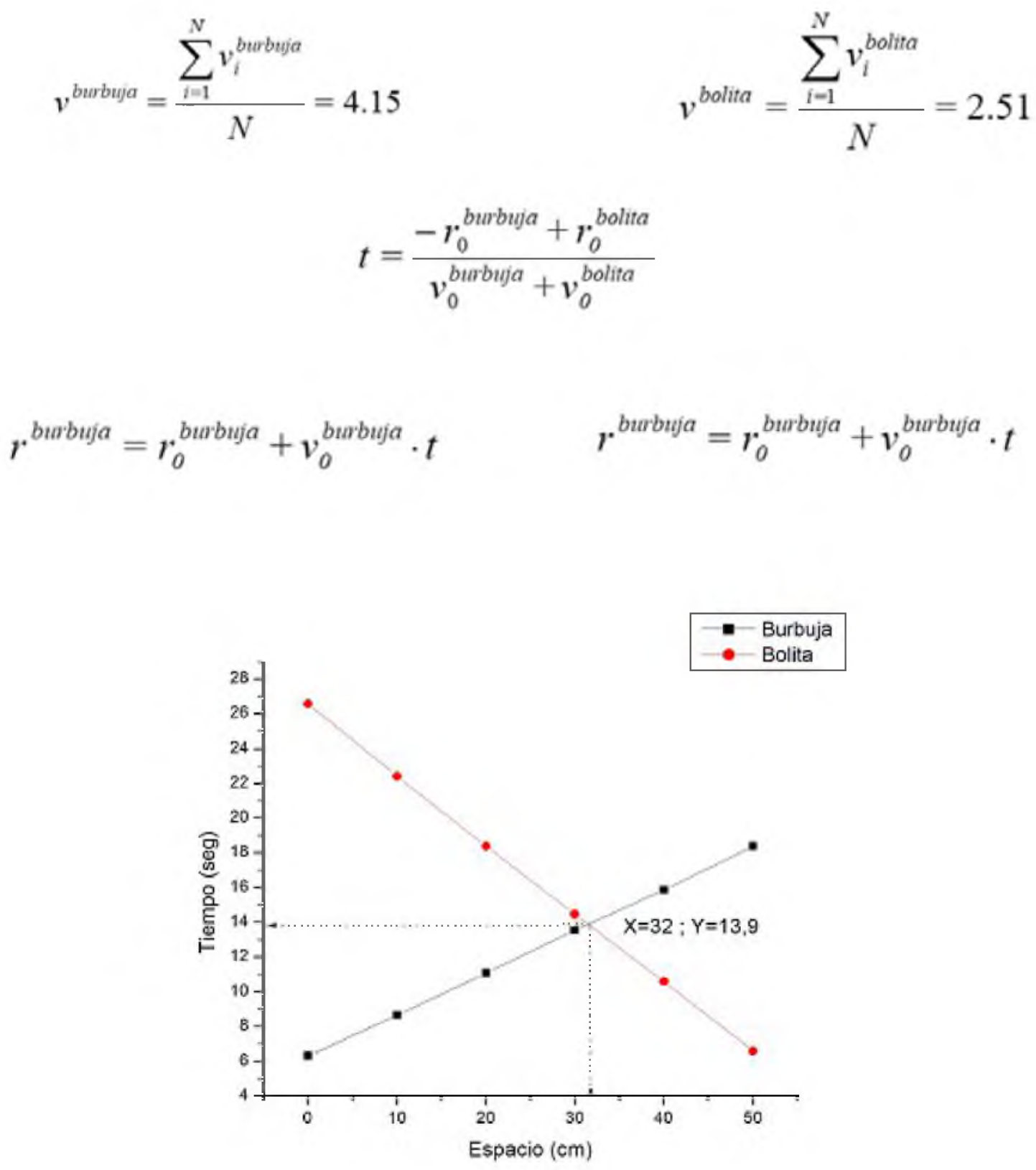

FIGURA 2. Se muestra gráfico de los movimientos de ambos móviles. Se marca la posición del punto de encuentro 
A. Secuencia del experimento
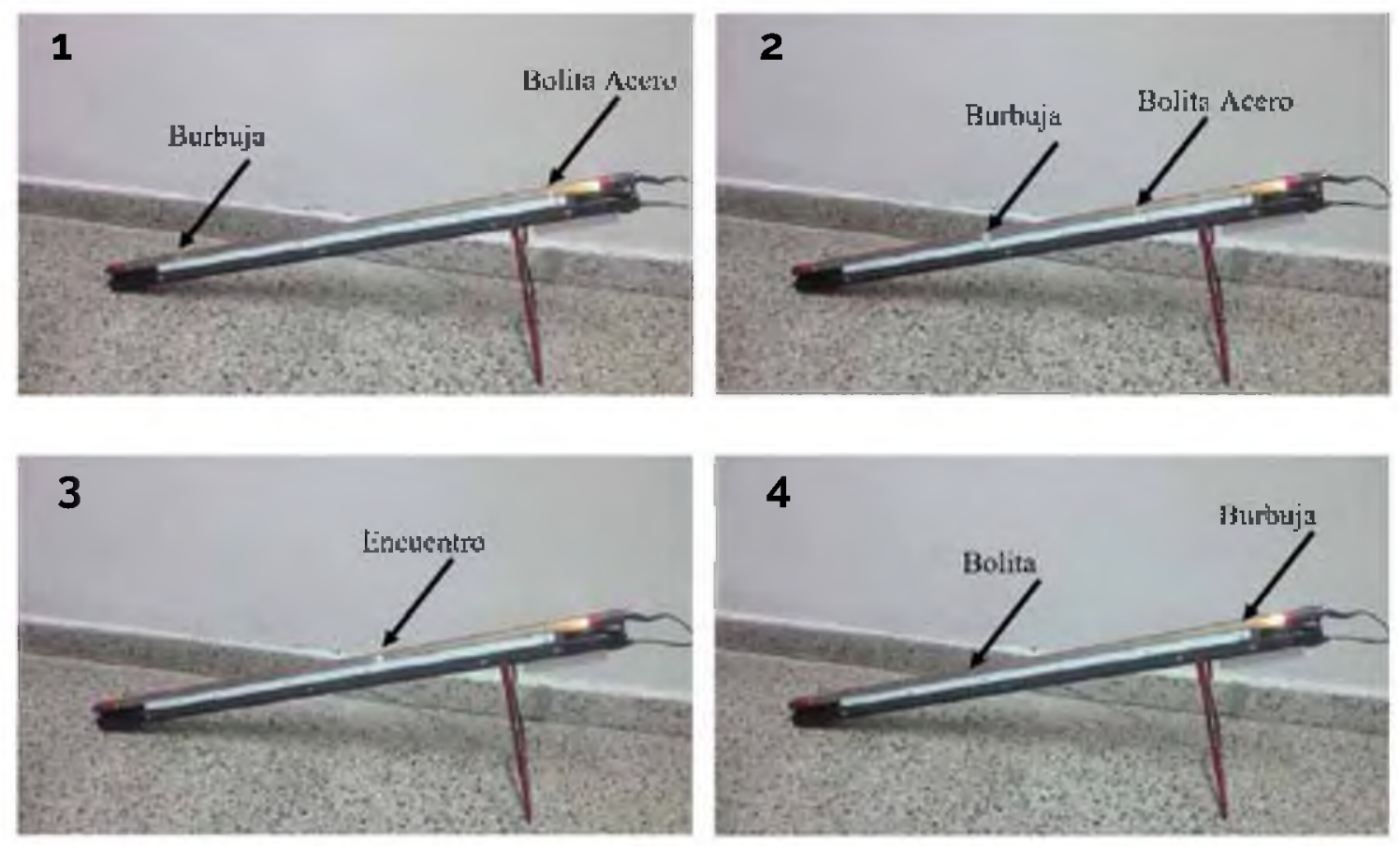

FIGURA 3. Se muestra secuencia fotográfica del experimento propuesto desde el inicio hasta el final pasando por el punto de encuentro de ambos móviles

\section{Conclusiones}

Durante las actividades realizadas en este laboratorio requerimos que, en adición a las mediciones mencionadas en el proceso experimental, los estudiantes documenten y reflexionen sobre su pensamiento. Esto permite el contraste entre el entendimiento previamente adquirido en forma empírica con esta nueva experiencia en un ambiente controlado y bajo la guía académica. Los MRU de los móviles pueden variar ligeramente al cambiar la viscosidad del líquido en la que se mueven y el ángulo de inclinación del mismo. En particular la última es la más sencilla de modificar. Esto produce puntos de encuentro diferentes. Con lo que cada estudiante o grupo de estudiantes debe enfrentar, necesariamente, la realización de su propio experimento con el mayor cuidado posible; que a su vez permite dar los primeros pasos en la forma de "pensar y hacer" experimentalmente. 


\section{Agradecimientos}

Los autores agradecen a la Secretaría de Ciencia y Técnica de la Universidad Nacional del Nordeste por el apoyo otorgado para la realización de este trabajo mediante el subsidio correspondiente al proyecto de investigación Pl:17Foo8, Resolución CS Nº 966/17.

\section{Referencias}

Bartlett, A. A. (2005).Television, football, and physics: Experiments in kinematics. Phys Teach. 43. 393

Berryhill, E.; Herrington, D.: Oliver, K. (2016) Kinematics Card Sort Activity: Insight into Students' Thinking. Phys. Teach. 54. 541

De Luca, R.: Faella, O. (2017). An ideal freekick. Eur. J. Phys. 38014002

Falcão, A. E. G.; Gomes, R. A.; Pereira, J. M.; Coelho, L. F. S.; Santos A. C. F. (2009). Cellular Phones Helping To Get a Clearer Picture of Kinematics. Phys. Teach. 47, 167

Gabriel, P.: Backhaus, U. (2013). Kinematics with the assistance of smartphones: Measuring data via GPS - Visualizing data with Google Earth. Phys. Teach. 51, 246

Gowin, D. B. (1981). Educating. Ithaca and London: Cornell University Press.

Guidugli, S:; Fernández Gauna C.; Benegas, J (2005). Graphical Representations of Kinematical Concepts: A Comparison of Teaching Strategies. Phys. Teach. 43. 334

Kagan, M. (2013). Thinking Outside of the Rectangular Box. Phys. Teach. 51, 215

Klein, P.; Müller, A.; Kuhn, J. (2017). Assessment of representational competence in ki- nematics. Phys. Rev. Phys. Educ. Res. 13. 010132

Kranjc, T.; Razpet, N. (2011). A School Experiment in Kinematics: Shooting from a Ballistic Car. Phys. Teach. 49, 442

Lowe, G. W: Ayars, E. (2010). Measuring the Flight Speed of Fire Bombers from Photos: An In-Class Exercise in Introductory Kinematics. Phys. Teach. 48, 106

Nelson, J. H. (1983). Kinematics of a student. Phys. Teach. 21, 386

Nordine, J. C. (2011). Motivating Calculus-Based Kinematics Instruction with Super Mario Bros. Phys. Teach. 49. 380

Rich, N. H. (1989). Bicycle computers and kinematics. Phys. Teach. 27, 627

Sathe, D. V. (1979). An aid for kinematics. Phys. Teach. 17.536

Stroink, G.; Guptill, E.; March, R.; (2003). The straight-line kinematics of a student, a fun demonstration. Phys. Teach. 41, 435

Vučeljić, M.; Šuškačević, M. (2016). Achievements of Montenegrian high-school students in Tugk test (test of understanding graphs - kinematics). AIP Conference Proceedings 1722,310007 